\title{
Computed Tomography from a Single Grating X-Ray Interferometer at a Laboratory Liquid-Metal-Jet Source
}

\author{
Andreas Balles ${ }^{1, *}$, Dominik Müller ${ }^{1}$, Jonas Dittmann ${ }^{1}$, Christian Fella ${ }^{2}$, Randolf Hanke ${ }^{2}$ and Simon \\ Zabler $^{2}$ \\ 1. Chair of X-Ray Microscopy, University of Würzburg, Germany \\ 2. Fraunhofer Institute for Integrated Circuits, NCTS group, Würzburg, Germany \\ * andreas.balles@physik.uni-wuerzburg.de
}

Grating interferometry is a well-established method in the field of X-ray imaging. An interferometer setup usually consists of two (Talbot interferometer) or three (Talbot-Lau interferometer) gratings. The data of such setups is recorded in a phase stepping mode by which three contrasts modes are retrieved from a sinusoidal curve, i.e. absorption, differential phase contrast (DPC) and darkfield image contrast (DIC). A decade ago, Takeda et al [1] reported on a more compact type of interferometer at a synchrotron beamline using a single (phase) grating by resolving the interference pattern - due to the Talbot effect - with a high resolution detector. This design allows to omit the phase stepping procedure resulting in an easy-to-handle setup with shorter exposure times. Since then, such setups have been developed at synchrotron and laboratory facilities. However, the latter consisted of gratings with large periods $(127 \mu \mathrm{m})[2,3]$ and $30 \mu \mathrm{m}$ pixel size compared to their synchrotron counterpart (period: several microns [4,5], pixel: $1 \mu \mathrm{m}$ and less). Gratings with a smaller period require both a high resolution detector and a sufficiently coherent source.

Here we show a computed tomography recorded at our laboratory single grating interferometer described previously in Ref. [6]. The setup combines a liquid metal jet source (95\% gallium) with a high resolution detector (pixel size $0.62 \mu \mathrm{m}$ ) leading to a single grating setup on the micrometer scale. As was shown by Thüring et al [7], such a source provides the required partial coherence, which allows to omit the source grating [8]. A sketch of the experimental setup is shown in Fig. 1. A $\pi / 2$ phase shifting grating made of $S U-8$ photoresist with a period of $\mathrm{p}=2.4 \mu \mathrm{m}$ is placed between the sample and the detector plane. The height of the grating complies the phase shift for the design energy of $9.25 \mathrm{keV}\left(\mathrm{K}_{\alpha}\right.$ emission line of gallium). The X-ray spot size was set to approx. $7 \mu \mathrm{m}$ FWHM. The detector system was an LSO:Tb scintillator optically connected to an sCMOS camera (Andor Zyla) with an effective pixel size of $0.62 \mu \mathrm{m}$. Four pixels approx. correspond to the grating period, including the cone beam magnification of 1.17.

The data processing of a single grating setup is identical to that of a conventional grating interferometer (evaluation of phase stepping curves), except for the fact that the phase stepping curves are generated from the recorded images [6]. A different method for treating the raw data with no constrains on the pixel to period ratio was proposed by Wen et al [2]. For the CT reconstruction, the filtered back projection technique was used by applying a Hilbert filter to the DPC images (avoiding numerical integration) and an empirically designed filter for the DIC in order to counter noise in the DIC volume [9]. The CT scans shown here were acquired with a total exposure time of $20 \mathrm{~h}$ with 497 angular projections.

We chose two samples to demonstrate the capabilities of DPC and DIC volume imaging and additionally compare the results of the single grating interferometer to measurements of a Talbot interferometer (same setup except for the additional analyzer grating; total exposure time 40h). The comparison of the reconstructed DPC volume (axial slice) of a piece of bacon is shown in Fig. 2. Obviously, the result from the single grating interferometer (top) shows fewer details than the slice of the Talbot interferometer 
(bottom). Nevertheless, it is possible to distinguish between fat and muscle tissue in both images. Figure 3 shows the comparison of the DIC of an aspirin pill crumb. As can be seen from the images, the majority of the signal comes from cracks in the sample representing the interfaces of the granular structure of the pill. The image quality in the reconstructed DIC volume of the single grating interferometer (top) suffers a lot from noise.

Concerning the DIC image of the single grating CT scan, the higher noise is a consequence of the reduced exposure time (factor 2 ) and visibility. The latter was $\mathrm{V} \approx 8.6 \%$ compared to a visibility of $\mathrm{V} \approx 24 \%$ in the interferometer setup. This reduction is caused by the detector MTF. Same applies to the DPC image that also suffers from increased noise and a less detailed information.

The results prove that we successfully demonstrated a single grating CT-setup on the micrometer scale in the laboratory. The detector was able to resolve the grating pattern but the visibility is clearly affected by its MTF. Hence, a longer exposure time would possibly counter the lower SNR. However, the less difficult handling of a single grating setup makes it an attractive alternative to Talbot interferometers. An improvement of the image quality could be achieved by a higher resolving detector and - more practical - by a second perpendicularly mounted phase grating representing a 2-D single grating setup.

\section{References:}

[1] Y Takeda et al, AIP Conference Proceedings (2007), p. 1361.

[2] HH Wen et al, Optics letters 35 (2010), p. 1932.

[3] E Bennett et al, Medical physics 37 (2010), p. 6047.

[4] KS Morgan et al, Optics letters 38 (2013), p. 4605.

[5] MC Zdora et al, Biomedical optics express 8 (2017), p. 1257.

[6] A Balles et al, AIP Conference Proceedings 1696 (2016), p. 020043.

[7] T Thüring et al, Applied Physics Letters 103 (2013), p. 091105.

[8] F Pfeiffer et al, Nature physics 2 (2006), p. 258.

[9] A Balles et al, Proc. SPIE 10391 Developments in X-Ray Tomography XI (2017), p. 1039109.

[10] We acknowledge funding of the Fraunhofer-Projektgruppe "NCTS" by the free state of Bavaria.

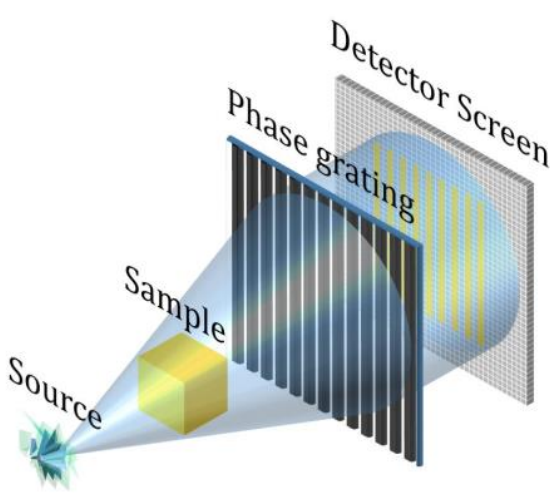

Fig 1. Sketch of the single grating interferometer setup. The use of a high-resolution detector allows to omit the analyzer grating.

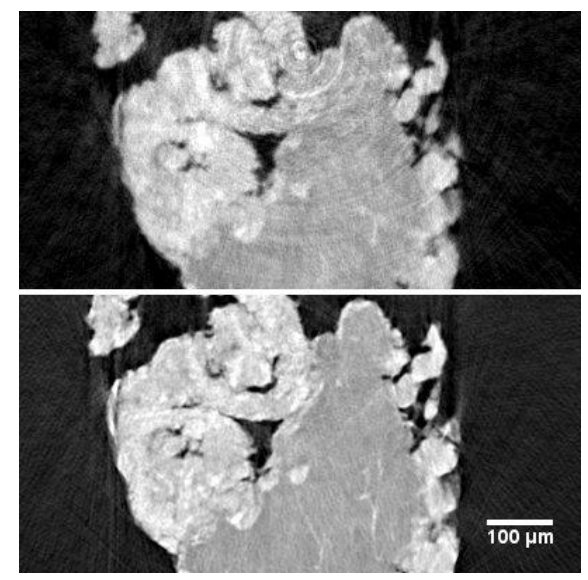

Fig 2. Reconstructed DPC slice from the single grating CT-scan of a bacon sample (top) compared to the interferometer scan (bottom).

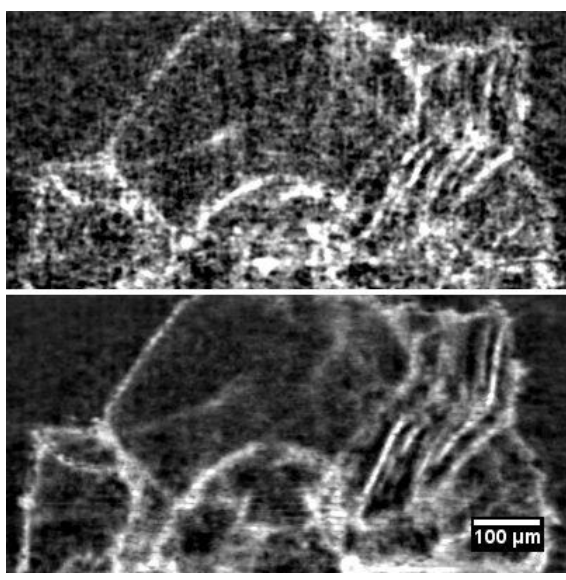

Fig 3. Reconstructed DIC slice from the single grating CT-scan of an aspirin pill (top) and from to the interferometer scan (bottom). 